\title{
Cataclysmic variables: New frontiers in multi-wavelength research
}

\author{
A. Odendaal* \\ University of the Free State, South Africa \\ E-mail: WinkA@ufs.ac.za \\ P. J. Meintjes \\ University of the Free State, South Africa \\ E-mail: MeintjPJ@ufs.ac.za
}

\begin{abstract}
Compact binary systems in which a white dwarf (WD) accretes material from a non-degenerate companion, constitute some of the most prevalent, yet intriguing astronomical objects in the universe. Of special interest is a class of these binaries known as cataclysmic variables (CVs), where the transfer of material from a red dwarf companion to the WD instigates a wide range of energetic processes causing transient, variable and eruptive behaviour. These binaries, as well as a closely related class of objects known as supersoft X-ray sources (SSSs) are therefore fascinating cosmic laboratories in which the effects of fundamentally important physical phenomena like turbulence, magnetic fields, gravitation, viscosity, and particle acceleration can be studied, by modelling the observed electromagnetic radiation, from the low energy radio waves all the way up to gammaray energies. Several CVs and SSSs have been shown to exhibit high-velocity outflows, many of which are expected to be driven by magnetic fields, and modern instruments have recently shown that these outflows are associated with radio and gamma-ray emission. These developments open up an exciting new area in this field. The upcoming commissioning of MeerKAT, as well as the newly available spectropolarimetric mode on SALT, in combination with international high-energy facilities, makes this the ideal time to undertake studies of the non-thermal emission in CVs in the form of synchrotron radio emission, and non-thermal optical emission, as well as potential particle acceleration yielding high energy emission.
\end{abstract}

4th Annual Conference on High Energy Astrophysics in Southern Africa

25-27 August, 2016

Cape Town, South Africa

${ }^{*}$ Speaker. 


\section{Introduction}

Population studies indicate that at least half of the stellar objects in the Universe are members of binary systems (e.g. [1, 2]). If the initial masses of the binary components are different, their evolution will occur on different time-scales, and one of the stars will evolve into a compact object, i.e. a white dwarf (WD), neutron star or black hole, before its companion. Compact binaries containing a WD with a non-degenerate companion are the most common interacting binaries, and include a wide variety of systems which can be referred to as cataclysmic variable-like binaries (see e.g. the review of [3]).

Cataclysmic variables $(\mathrm{CVs})$ are compact binary systems $\left(\sim 1 \mathrm{R}_{\odot}\right)$ containing a WD primary accreting from a red dwarf companion (see [4] for a detailed treatment of CVs). An accretion disc is usually formed around the WD. The disc is highly luminous in the optical and ultraviolet wavebands due to the release of gravitational potential energy. In the case of "non-magnetic" CVs (WD magnetic field $B<10^{6} \mathrm{G}$ ), the disc extends down to the WD surface. However, in the case of magnetic CVs, the disc structure is disrupted to varying degrees by the stronger WD magnetic field: In intermediate polars $\left(10^{6} \mathrm{G} \lesssim B \lesssim 10^{7} \mathrm{G}\right)$ the disc is disrupted at the magnetospheric radius $R_{m}$, and at radii smaller than $R_{m}$, accretion takes place along the magnetic field lines to the WD polar caps. In polars $\left(B>10^{7} \mathrm{G}\right)$, no disc is formed, and the accretion stream impinges directly onto the WD magnetosphere.

$\mathrm{CVs}$ are characterized by spectacular brightness changes, which are a direct result of the dynamic nature of the accretion process. Novae represent the most dramatic events, associated with brightness increases ranging from 6 to 19 magnitudes. Nova outbursts constitute runaway nuclear burning of hydrogen-rich material accreted on the WD surface. For "classical novae" $(\mathrm{CNe})$, only one eruption has been observed, while for "recurrent novae" (RNe), multiple outbursts have already been observed. During the outburst, a fraction of the accreted material is blown off the WD (see Fig. 1). The decline time for the various classes of novae are (e.g. [4], p. 263): Very fast $(<10 \mathrm{~d})$, Fast (10-25 d), Moderately fast (26-80 d), Slow (81-150 d) and Very slow (151-250 d).
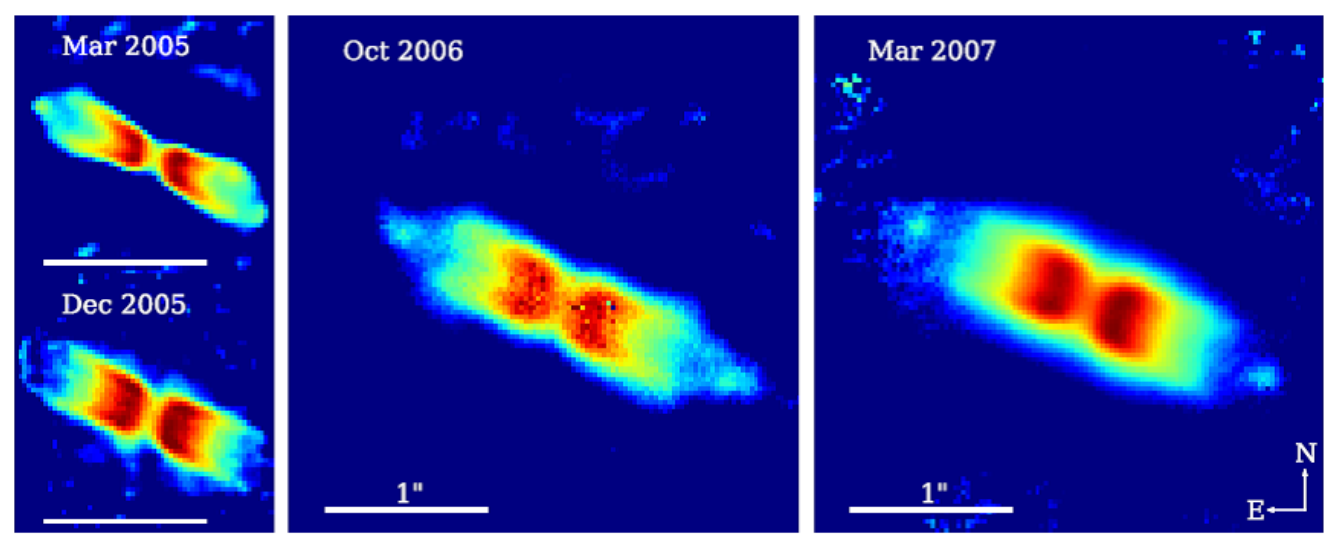

Figure 1: Evolution of the nova eruption in V445 Puppis over a two year period, measured with the NAOS/CONICA on the VLT. Clearly visible is a bipolar outflow. Adopted from [5].

Outbursts with much smaller amplitudes (about 2 to 5 magnitudes) are observed in dwarf novae 
(DNe). DN outbursts are not caused by thermonuclear burning, but are the result of a thermalviscous instability in the disc surrounding a weakly magnetized WD. CVs also include a group that is known as nova-like variables or "novalikes", which are high accretion rate CVs (see below), in which no large eruptions as in novae or DNe have yet been observed. This class therefore includes CVs in pre- or post-novae states. The typical mass transfer rates encountered in CVs range from $\sim(2-50) \times 10^{-11} \mathrm{M}_{\odot} \mathrm{yr}^{-1}$ in DNe during quiescence, to $\sim(3-10) \times 10^{-9} \mathrm{M}_{\odot} \mathrm{yr}^{-1}$ in nova remnants, novalikes and DNe during outburst (e.g. [4, pp. 64-66]). The typical luminosity of a nova remnant or novalike is $\sim 10^{33} \mathrm{erg} \mathrm{s}^{-1}$, and is derived from the release of gravitational potential energy of material transferred from the companion undergoing accretion onto the WD.

During the last few decades, another class of WD binaries closely related to CVs has been established, i.e. supersoft X-ray sources (SSSs, see [6] for a review). The defining observational properties of this class are (i) their extreme X-ray luminosities $\left(\sim 10^{36}\right.$ to $\left.10^{38} \mathrm{erg} \mathrm{s}^{-1}\right)$, and (ii) the extreme softness of their X-ray spectra, typically with effective temperatures of $\sim 20-100 \mathrm{eV}$. The vast majority of observed SSSs are binary systems. It was shown that their X-ray properties can be explained by steady nuclear burning of accreted hydrogen on the surface of a WD [7]. To sustain persistent hydrogen nuclear burning on the WD surface, the mass transfer rate in SSSs needs to be significantly higher than in CVs (approximately $10^{-7} \mathrm{M}_{\odot} \mathrm{yr}^{-1}$ ) (see Fig. 2).

In $\mathrm{CVs}$, the WD is more massive than the donor. However, the high accretion rate required to drive persistent surface nuclear burning in SSSs is only possible if the donor is more massive than the WD. The main characteristics of SSSs distinguishing them from CVs are therefore their inverted mass ratios, high accretion rates and extreme luminosities derived from surface nuclear burning. In SSSs the transfer of mass from a non-degenerate companion to the WD can give new life to the WD. This interaction results in the occurrence of a rich plethora of energetic phenomena, providing a cosmic laboratory for studying fundamental physical processes related to e.g. gravitation, viscosity and magnetic fields, as well as the associated radiation fields, under extreme conditions. There are several hundred known SSSs, and an even greater number of CVs. These sources are very close to us in astronomical terms, and therefore appear bright enough for detailed multi-wavelength studies. Magnetohydrodynamic (MHD) turbulence and its accompanying influence on accretion and radiation represent phenomena that are encountered in many different types of Galactic and extragalactic accretion-driven systems, including active galactic nuclei (AGN). Constraining important parameters for WD binaries therefore has a broad impact, since these results can be extrapolated to other systems.

WD binaries have proven themselves to be truly multi-wavelength sources, and several intriguing observational discoveries in these systems have been made during recent years, ranging from radio to gamma-ray energies. In this short review, we do not attempt to present a comprehensive summary of all multi-wavelength advances in the field, but rather aim to highlight some of the interesting results that are especially relevant for the research planned in our group for the next few years. Of special interest is the different types of observational evidence for outflows from the WD in these systems. In $\S 2$, a brief description of the key role that MHD turbulence is expected to play in accreting binaries is provided. As described in $\S 3$, evidence for outflows are present in the spectra of several sources, and are very likely to be linked to the presence of MHD turbulence. Recent radio and gamma-ray detections also indicate the presence of outflows, and are discussed in $\S 4$ and $\S 5$ respectively, followed by a brief summary of future prospects in $\S 6$. 


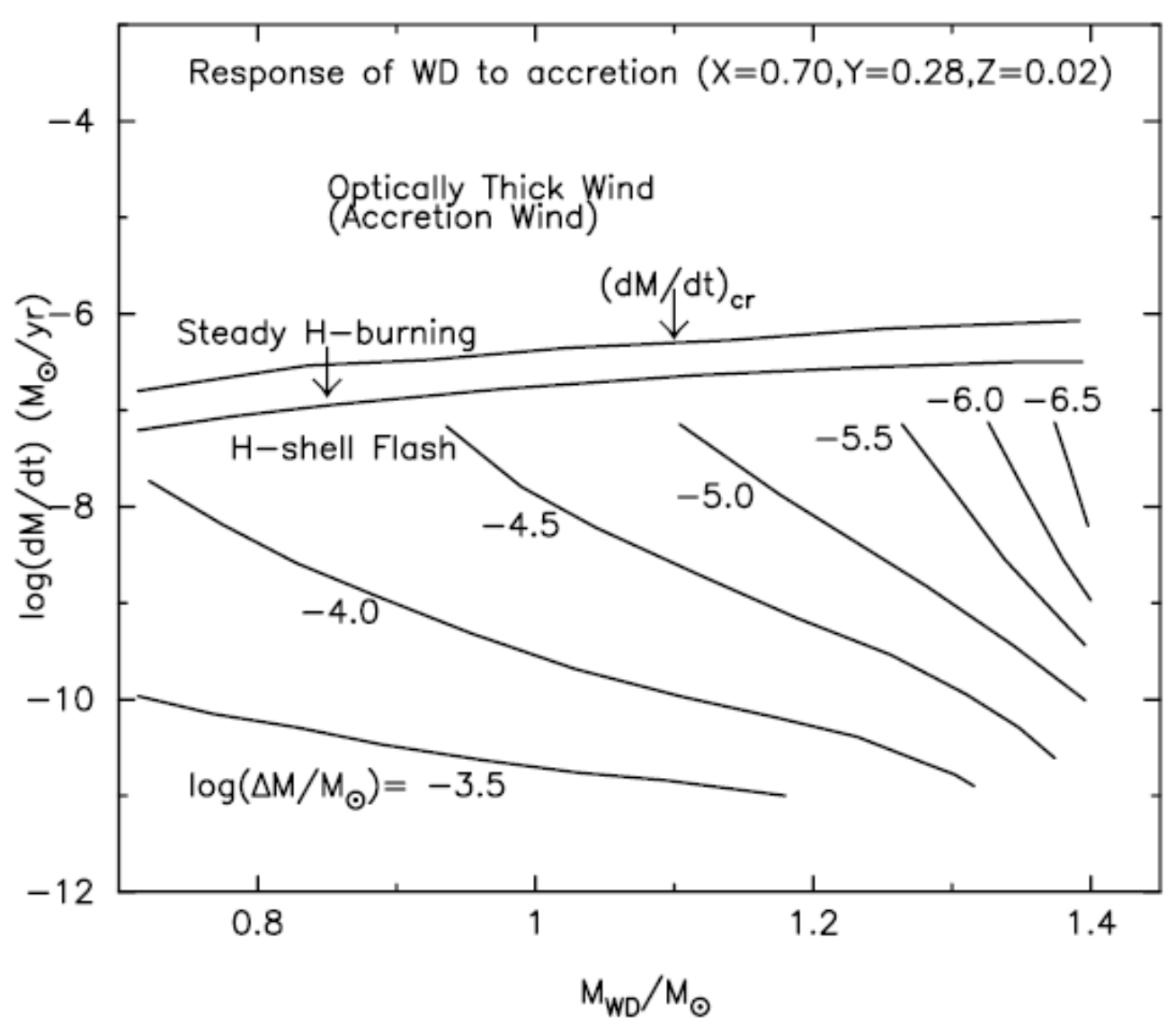

Figure 2: The required accretion rate onto the surface of a white dwarf to sustain steady nuclear burning. Adopted from [8].

\section{Magnetohydrodynamic turbulence}

During accretion in CVs and SSSs, material needs to lose angular momentum, thus angular momentum has to be transported outwards in the disc. Turbulent viscosity is expected to play a major role, since the effect of molecular viscosity is not strong enough. Although hydrodynamic turbulence should be able to aid in the outward angular momentum transfer in the disc, hydrodynamic instabilities will be smoothed out on the dynamical time-scale of the disc [9]. It has also been shown that convective turbulence does not provide an effective mechanism of outward angular momentum transport in the disc [10].

A theory of turbulence driven by magnetohydrodynamic (MHD) instabilities was developed [11], which provides a sustainable mechanism of angular momentum transport in a disc. The accretion discs in compact binaries are hot enough to be significantly or fully ionized. Magnetic field lines are expected to thread the disc, either due to the WD magnetic field, or possibly fields advected from the secondary star. Considering the close connection between electricity and magnetism, there will be a strong coupling between the disc material and the magnetic fields. Charged particles can readily flow along magnetic field lines, but can not easily cross them, therefore the 
magnetic field lines can be considered to be "frozen" into the plasma.

Consider two fluid elements at different disc radii, connected by a magnetic field line. The element at smaller radius $R$ has a higher angular velocity than the outer element, causing the field line to become "stretched", almost like a slinky. This causes a braking of the inner element, and an acceleration of the outer element, transferring momentum from the inner to the outer element. Consequently the inner element moves into an orbit even closer to the primary with a higher angular velocity, with the outer element moving into a wider orbit with a lower angular velocity. This stretches the field line even more. The stretching of the field line is equivalent to strengthening the field, so the initial fields are amplified (see Fig 3). This is known as the magnetorotational instability (MRI) [11, 12].

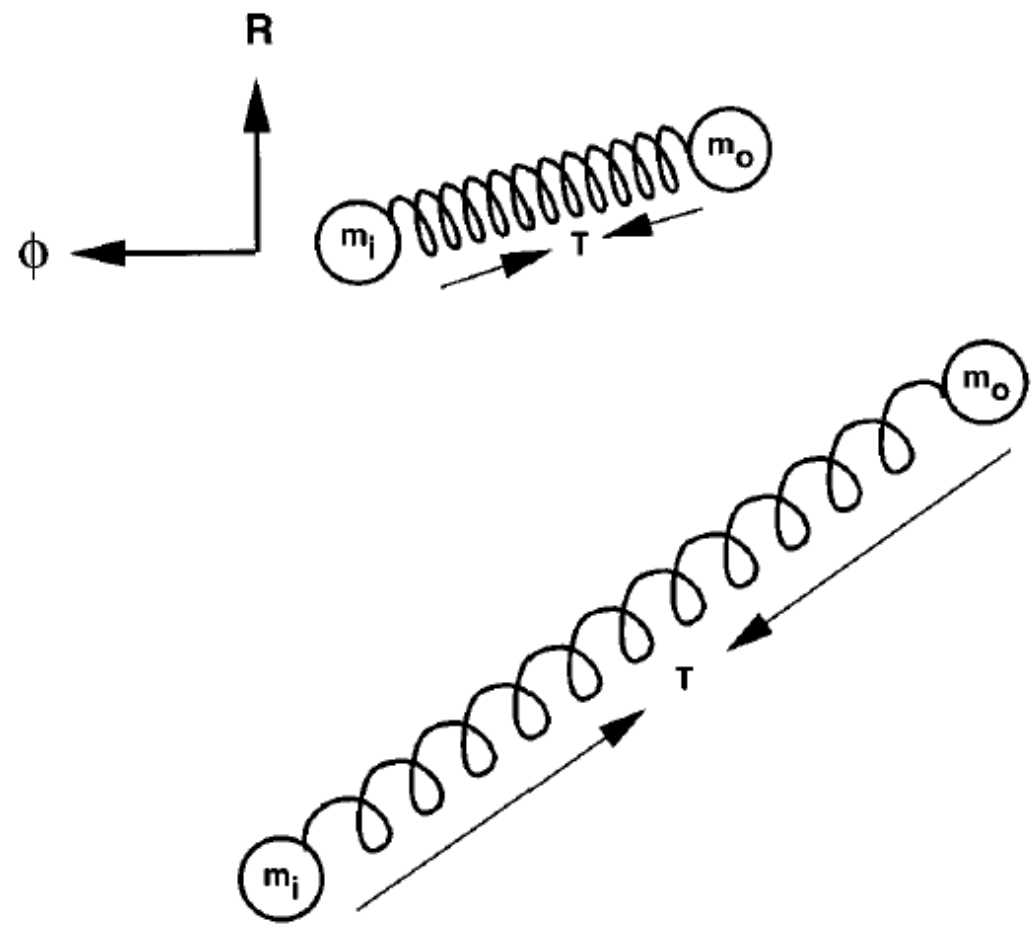

Figure 3: Two mass elements $m_{\mathrm{i}}$ and $m_{\circ}$ are connected via a weak spring which exerts a tension force T, orbiting a compact object at a radial distance R. Through the exchance of angular momentum via the MRI the inner mass $m_{\mathrm{i}}$ is spiralling inward, while the orbit of the outher mass $m_{\circ}$ is pushed to larger radii. Adopted from [12].

As the field line is increasingly stretched, reconnection will ultimately take place to dissipate energy, resulting in "bubbles" of gas being transported to different radii, and the ordered Keplerian flow breaking up into turbulence. This instability develops rapidly and is very powerful, although the magnetic field should not be too strong, i.e. the magnetic field energy density must be less than the thermal energy density. It has also been shown that the existing magnetic fields in the disc are amplified by the presence of turbulence through the dynamo effect (e.g. [13, pp. 211-248]). 


\section{Spectral evidence of outflows}

Outflows are generally divided into two classes: winds and jets. The distinction is not always clear, but "jets" generally refer to narrow or well collimated outflows propagating in a direction more or less perpendicular to the disc surface, with terminal velocities approximately equal to the escape velocity of the central object. Winds, on the other hand, are not so well collimated, and exhibit velocities ranging from approximately $10 \%$ to $100 \%$ of the WD escape velocity, $v_{\text {esc }}$ [14]. Evidence for the presence of outflows in the form of Doppler shifted emission features and P Cyg absorption features has been found in the optical spectra of 4 supersoft X-ray binaries $[15,16,17,18,19]$. The main Balmer lines of the galactic sources RX J0019.8+2156 and MR Vel, as well as the Large Magellanic Cloud (LMC) source RX J0513.9-6951, exhibit P Cyg absorption profiles, together with sharp Doppler-shifted emission features, which are ascribed to emission from collimated jets. CAL 83 in the LMC also exhibits P Cyg profiles, but with broad, variable Doppler shifted emission structures that are expected to come from an outflow with a larger collimation angle.

Evidence for winds in CVs have been found in the ultraviolet (UV), extreme UV and X-ray bands, but mostly in the form of P Cyg profiles in the UV, presumably originating from biconical, rotating winds (e.g. [20] and references therein). However, evidence of collimated jets in CVs have until recently been notoriously elusive (e.g. [21]). Triple $\mathrm{H} \alpha$ emission lines have been observed in the RN T Pyx, but this source is similar to SSSs in the sense that hydrogen burning is still taking place on the WD surface as part of a slow nova outburst, even though its accretion rate is lower than in SSSs [22]. Bipolar collimated outflows have also recently been observed in optical data of the CN V445 Pup [5].

The inferred outflow velocities in the SSSs and CVs above are several thousand $\mathrm{km} \mathrm{s}^{-1}$, of the same order as $v_{\text {esc }}$. These outflows may be radiation-driven, i.e. either continuum-driven, where momentum is transferred from continuum photons to the material, or more likely line-driven, where momentum transfer takes place via line opacity (e.g. [23]). However, it is also interesting that the formation of magneto-centrifugally accelerated winds or jets from an accretion disc threaded by a weak vertical magnetic field is a natural consequence of the existence of the MRI, the latter being an almost essential element of disc accretion [24]. It is therefore highly likely that magneto-centrifugal acceleration plays a significant role in the production of the outflows in SSSs and CVs.

\section{Non-thermal emission}

When charged particles are accelerated in a magnetic field, polarized radiation with a characteristic spectral energy distribution is produced (e.g. [25, pp. 255-273]). If the motion of the charged particles is ultra-relativistic, the non-thermal emission is known as synchrotron radiation, which is often observed in radio jets. In jet regions, electrons can potentially be accelerated by shocks to relativistic velocities. In the non-relativistic case, the non-thermal radiation is referred to as cyclotron radiation. Outflows provide ideal environments in which to observe non-thermal radiation, due to their low particle density and associated absorption.

The existence of radio jets has been proven during certain states of black hole and neutron star binaries (e.g. the reviews of $[26,27]$ ). Synchrotron radio emission has also been detected from the 
jets of some massive protostars (e.g. [28]). However, very few CVs have been well studied at radio energies. In the 1980s, three non-magnetic CVs were detected in the radio: SU Uma, EM Cyg and TY Psc [29, 30, 31]. The radio-detected magnetic CV sample was also very small, consisting of AM Her, V834 Cen, ST Lmi, AR UMa, AE Aqr, DQ Her and BG Cmi (e.g. [32, 33, 34, 35]). However, at the time of these discoveries there were also a large number of non-detections.

However, with the better sensitivities of modern radio detectors, radio observations of CVs is becoming a new and intriguing topic in this field. Bipolar collimated outflows were observed in VLA and VLBA radio images of the RN RS Oph [36]. The prototypical DN SS Cyg was observed with various facilities to exhibit reproducible radio flares during its recent outbursts ([37] and references therein). The VLA detections of the DNe RX And, U Gem, Z Cam, SU UMa and $\mathrm{YZ}$ Cnc established that $\mathrm{DNe}$ as a class are indeed radio emitters during outburst [38]. Radio detections of four novalikes with the VLA have also been reported: V3885 Sgr [39], RW Sex, V603 Aql and TT Ari [40].

The overall range of spectral indices for a power law fit $\left(F_{v} \propto v^{\alpha}\right)$ reported by Coppejans et al. are $-2.9 \pm 1.5$ to $4.2 \pm 1.3$ for the DNe [38], and $-0.5 \pm 0.7$ to $1.7 \pm 0.8$ for the novalikes [40]. These are compatible with non-thermal synchrotron emission models, and consistent with emission from radio jets. These authors also showed that, assuming reasonable parameters for these sources, the brightness temperatures are in the $10^{9}$ to $10^{12} \mathrm{~K}$ range, and such high brightness temperatures also represent strong evidence for the non-thermal nature of the radio emission. This is important since it implies particle acceleration in magnetic outflows.

However, to firmly establish these findings, and to integrate these new developments into the existing models for CV-like systems, a larger sample of radio detected CVs are needed. The current construction of MeerKAT (and later the SKA) places South Africa in an excellent position to contribute enormously in this regard. In fact, the study of radio emission from accreting WD binaries is one of the 5 focus areas of ThunderKAT (The HUNt for Dynamic and Explosive Radio transients with MeerKAT), one of the Large Survey Projects planned for MeerKAT. For the classical binary SSSs, no radio detections have been reported yet, only upper limits [41]. Since there is clear evidence for outflows in the optical spectra of several SSSs, the prospect of detecting radio jets in these systems for the first time with MeerKAT is very exciting.

Polarimetric studies in the optical can also further enhance our understanding of these systems. In addition to the availability of the HIgh speed Photo-POlarimeter (HIPPO) on the South African Astronomical Observatory (SAAO) 1.9-m telescope [42], the recent re-commissioning of the spectropolarimetric mode of the Robert Stobie Spectrograph (RSS) on Southern African Large Telescope (SALT), see [43]) opens up the possibility of performing detailed polarization studies on interesting transient $\mathrm{CV}$ candidates. The detection of linearly polarized light during DN flares can indicate synchrotron emission extending to optical wavelengths. This will provide crucial information regarding the magnetic field geometry, the magnetic field strength in the outflow, and also the particle acceleration energies.

In addition to the detection of synchrotron emission, there is also the possibility of observing polarized optical cyclotron emission from regions closer to the WD polecaps, where non-relativistic electrons interact with the WD magnetic field. Longslit spectra from the RSS can also be used to search for Doppler-shifted features associated with jets (e.g. [19]). Polarization studies of SSSs would also be very interesting. However, due to the observed SSS sample being mostly extra- 
galactic, and the WD envelope being very extended, it may be difficult to detect potential polarized cyclotron emission from these systems.

\section{Particle acceleration and high energy emission}

Several novae have recently been observed as $>100 \mathrm{MeV}$ gamma-ray sources by the FermiLarge Area Telescope (Fermi-LAT), starting with the symbiotic nova V407 Cyg [44] and followed by the CNe V1324 Sco, V959 Mon and V339 Del [45], V1369 Cen 2013 and V5668 Sgr 2015 [46].

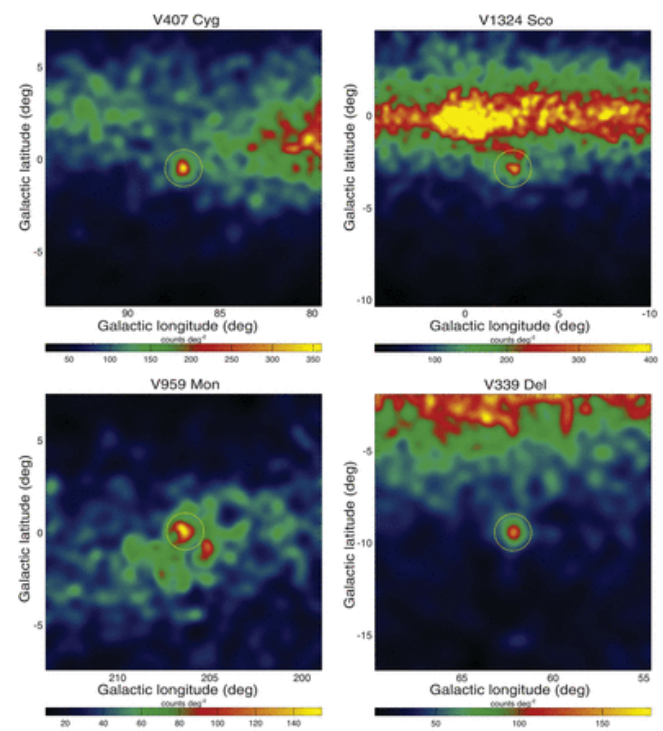

Figure 4: Fermi-LAT skymaps showing the gamma-ray detections of 4 Novae systems. Adopted from [47].

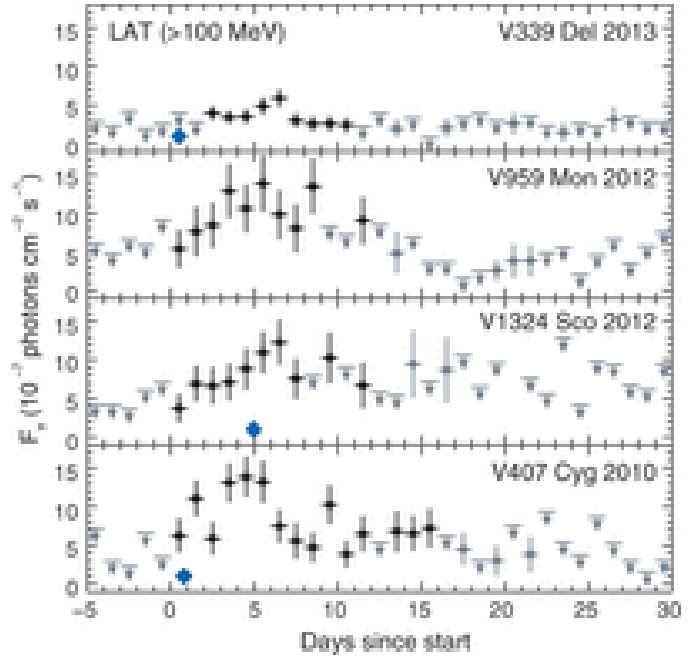

Figure 5: Gamma-ray lightcurves of the 4 Novae systems above $100 \mathrm{MeV}$. Adopted from [47].

Two plausible mechanisms for the gamma-ray production have been discussed[45]: In the hadronic scenario, high-energy protons in the nova ejecta interact with nuclei (either in the circumstellar wind of a red giant companion in the case of symbiotic novae, or the surrounding interstellar medium for classical novae). This interaction results in the formation of neutral pions, each of which decays into two gamma-ray photons. In the leptonic scenario, gamma-rays are produced when accelerated electrons interact with low-energy photons through inverse Compton scattering, or with surrounding atoms through Bremsstrahlung.

However, it has been shown that the hadronic model for gamma-ray production is much more likely, due to the low inferred electron acceleration efficiency in the leptonic model [48]. Gamma-ray novae are of high importance, since they present a view of particle acceleration at non-relativistic shocks, and multi-wavelength campaigns during gamma-ray flares can be used to constrain the shock parameters [48]. Therefore, in order to distinguish between the different models, and to place better constraints on the model parameters, more observations of these transient gamma-ray events are needed.

The detection of gamma-rays from nova ejecta poses the very interesting possibility of the detection of gamma-ray emission from SSSs for the first time. In these sources, the acceleration 
of particles in the high-velocity jets may also yield gamma-ray emission, and such detections can provide fundamentally important information on the energetics of the jet launching mechanism. As explained above, one would expect gamma-ray emission from those sources where there is enough material surrounding the source (e.g. from previous ejecta, or the interstellar medium) to interact with the accelerated particles. An infrared excess observed in some CVs could be indicative of dusty circumbinary material as a result of mass loss (e.g. [49]), which could be a potential way to identify sources where target material for neutral pion production may be present. One should also investigate possible gamma-ray emission from novalikes and DNe where evidence for jets have been found in radio and optical wavebands, since gamma-ray emission has been detected in similar non-relativistic outflows from the radio jets of the massive protostar IRAS 18162-2048 [50].

\section{Future Prospects}

During recent years, observational discoveries in the field of cataclysmic variables (CVs) and the related class of supersoft X-ray sources (SSSs) have opened up some interesting new frontiers in multi-wavelength research for these systems. In addition to existing spectral evidence for outflows in the optical through X-ray wavebands, recent radio detections of CVs are consistent with synchrotron emission from radio jets. The construction of MeerKAT and eventually the SKA provides the exciting prospect of observing radio jets in SSSs for the first time, and to increase the number of radio-detected CVs, enhancing our understanding of the launching mechanisms and relation with other source properties.

Gamma-ray detections from nova ejecta raise the interesting possibility of the detection of high energy photons from energetic outflows in other WD binaries. Surveys like the Catalina Real-time Transient Survey (CRTS), the Centre for Backyard Astrophysics (CBA), the American Association of Variable Star Observers (AAVSO) and the Palomar Transient Factory can be used to identify sources that exhibit regular outbursts in the optical, making them promising candidates for associated high-energy outbursts. In addition to exploring detections of new outbursts with Fermi-LAT, the Swift gamma-ray burst monitor, the International Gamma-Ray Astrophysics Laboratory (INTEGRAL) and the Italian gamma-ray mission, the Astro-Rivelatore Gamma Immagini Leggero (AGILE), the archives of older gamma-ray telescopes on-board the Compton Gamma-Ray Observatory (CGRO) can also be investigated. With our current knowledge of novae and other transient events in WD binaries during the last decades, these archives can be searched to check whether any of the unidentified gamma-ray detections were possibly associated with transient WD binary activity. The unprecedented sensitivity of the soon-to-come Cherenkov Telescope Array (CTA) is also expected to lead to many new discoveries in the field [51].

As part of the hadronic process, the decay of charged pions and muons can also be associated with neutrino production. The possible detection of neutrinos from eruptive WD binaries by detectors such as IceCube, combined with data from the IMB neutrino detector, constitutes a fascinating future follow-up for this project, extending the "multi-wavelength" study to a truly "multi-messenger" approach (see also the remarks of [52]). 


\section{Acknowlegements}

The principle author expresses her appreciation towards the Scientific Organizing Committee (SOC) for the invitation to present this review talk at HEASA2016. Both authors express their sincere appreciation towards the Local Organizing Committee (LOC) for the arrangements, the South African Astronomical Observatory (SAAO) for hosting HEASA2016 on very short notice as well as DST/NRF for funding it through SA-GAMMA.

\section{References}

[1] W. D. Heintz, A Statistical Study of Binary Stars, JRASC 63 (1969) 275.

[2] T. J. Herczeg, Double stars - The closest and the most distant systems, in IAU Colloq. 69: Binary and Multiple Stars as Tracers of Stellar Evolution (Z. Kopal and J. Rahe, eds.), vol. 98 of Astrophysics and Space Science Library, pp. 145-151, 1982.

[3] E. P. J. Van den Heuvel, The Formation and Evolution of Relativistic Binaries, in Astrophysics and Space Science Library (M. Colpi, P. Casella, V. Gorini, U. Moschella, and A. Possenti, eds.), vol. 359 of Astrophysics and Space Science Library, p. 125, 2009.

[4] B. Warner, Cataclysmic Variable Stars. No. 28 in Cambridge Astrophysics Series. Cambridge University Press, Cambridge, 2 ed., 1995.

[5] P. A. Woudt, et al., The Expanding Bipolar Shell of the Helium Nova V445 Puppis, ApJ 706 (2009) 738-746.

[6] P. Kahabka and E. P. J. van den Heuvel, Super Soft Sources, ch. 11, pp. 461-474. Cambridge University Press, New York, 2006.

[7] E. P. J. Van den Heuvel, D. Bhattacharya, K. Nomoto, and S. A. Rappaport, Accreting white dwarf models for CAL 83, CAL 87 and other ultrasoft X-ray sources in the LMC, A\&A 262 (1992) 97-105.

[8] I. Hachiso, M. Kato, Recurrent novae as a progenitor for type Ia supernovae: I. RS Ophiuchi subclass: Systems with a red giant companion. , ApJ 558 (2001) 323-350

[9] S. A. Balbus, J. F. Hawley, and J. M. Stone, Nonlinear Stability, Hydrodynamical Turbulence, and Transport in Disks, ApJ 467 (1996) 76.

[10] J. M. Stone and S. A. Balbus, Angular Momentum Transport in Accretion Disks via Convection, ApJ 464 (1996) 364.

[11] S. A. Balbus and J. F. Hawley, A powerful local shear instability in weakly magnetized disks. I Linear analysis. II - Nonlinear evolution, ApJ 376 (1991) 214-233.

[12] S. A. Balbus and J. F. Hawley, Instability, turbulence, and enhanced transport in accretion disks, Reviews of Modern Physics 70 (1998) 1-53.

[13] C. G. Campbell, ed., Magnetohydrodynamics in Binary Stars, vol. 216 of Astrophysics and Space Science Library, 1997.

[14] N. Murray, Disk outflows; radiation or hydromagnetic driving?, in The Physics of Cataclysmic Variables and Related Objects (B. T. Gänsicke, K. Beuermann, \& K. Reinsch, ed.), vol. 261 of Astronomical Society of the Pacific Conference Series, p. 308, 2002.

[15] K. A. Southwell, M. Livio, P. A. Charles, D. O'Donoghue, and W. J. Sutherland, The Nature of the Supersoft X-Ray Source RX J0513-69, ApJ 470 (1996) 1065. 
[16] C. M. Becker, R. A. Remillard, S. A. Rappaport, and J. E. McClintock, Bipolar Jets and Orbital Dynamics of the Supersoft X-Ray Source RX J0019.8+2156, ApJ 506 (1998) 880-891.

[17] T. Tomov, U. Munari, D. Kolev, L. Tomasella, and M. Rejkuba, Jets from the galactic supersoft X-ray source RX J0019.8+2156, A\&A 333 (1998) L67-L69.

[18] C. Motch, The transient jet of the galactic supersoft X-ray source RX J0925.7-4758, A\&A 338 (1998) L13-L16.

[19] A. Odendaal, P. J. Meintjes, P. A. Charles, and A. F. Rajoelimanana, SALT observations of outflows in three supersoft X-ray binaries, Proceedings of Science PoS(SSC2015)071 (2015).

[20] C. S. Froning, Observations of Outflows in Cataclysmic Variables, in The Astrophysics of Cataclysmic Variables and Related Objects (J.-M. Hameury and J.-P. Lasota, eds.), vol. 330 of Astronomical Society of the Pacific Conference Series, p. 81, 2005.

[21] N. Soker and J.-P. Lasota, The absence of jets in cataclysmic variable stars, A\&A 422 (2004) 1039-1043.

[22] T. Shahbaz, M. Livio, K. A. Southwell, and P. A. Charles, T Pyxidis: The First Short-Period Cataclysmic Variable with a Collimated Jet, ApJ 484 (1997) L59-L62.

[23] D. Proga, Theory of Outflows in Cataclysmic Variables, in The Astrophysics of Cataclysmic Variables and Related Objects (J.-M. Hameury and J.-P. Lasota, eds.), vol. 330 of Astronomical Society of the Pacific Conference Series, p. 103, 2005.

[24] S. Fromang, H. Latter, G. Lesur, and G. I. Ogilvie, Local outflows from turbulent accretion disks, $A \& A 552$ (2013) A71.

[25] J. A. Irwin, Astrophysics: Decoding the Cosmos. Wiley-VCH Verlag, 2007.

[26] R. Fender, Jets from X-ray binaries, pp. 381-419. 2006.

[27] E. Gallo, Radio Emission and Jets from Microquasars, in Lecture Notes in Physics, Berlin Springer Verlag (T. Belloni, ed.), vol. 794 of Lecture Notes in Physics, Berlin Springer Verlag, p. 85, 2010.

[28] C. Carrasco-González, L. F. Rodríguez, G. Anglada, J. Martí, J. M. Torrelles, and M. Osorio, A Magnetized Jet from a Massive Protostar, Science 330 (2010) 1209.

[29] A. O. Benz, E. Fuerst, and A. L. Kiplinger, First detection of radio emission from a dwarf nova, Nature 302 (1983) 45.

[30] A. O. Benz and M. Guedel, VLA detection of radio emission from a dwarf nova, A\&A 218 (1989) $137-140$.

[31] K. C. Turner, $12 \mathrm{~cm}$ Observations of Stellar Radio Sources, in Radio Stars (R. M. Hjellming and D. M. Gibson, eds.), vol. 116 of Astrophysics and Space Science Library, p. 283, 1985.

[32] A. E. Wright, R. T. Stewart, G. J. Nelson, O. B. Slee, and M. Cropper, Detection of the AM HER type cataclysmic variable V834 CEN at radio wavelengths, MNRAS 231 (1988) 319-324.

[33] M. Abada-Simon, A. Lecacheux, T. S. Bastian, J. A. Bookbinder, and G. A. Dulk, The spectrum and variability of radio emission from AE Aquarii, ApJ 406 (1993) 692-700.

[34] P. E. Pavelin, R. E. Spencer, and R. J. Davis, A 5-GHZ Radio Survey of Magnetic Cataclysmic Variables, MNRAS 269 (1994) 779.

[35] P. A. Mason and C. L. Gray, AR Ursae Majoris Discovered to Be a Persistent Radio Polar: Results from a VLA Survey of Magnetic Cataclysmic Variables, ApJ 660 (2007) 662-668. 
[36] J. L. Sokoloski, M. P. Rupen, and A. J. Mioduszewski, Uncovering the Nature of Nova Jets: A Radio Image of Highly Collimated Outflows from RS Ophiuchi, ApJ 685 (2008) L137-L140.

[37] T. D. Russell, et al., The reproducible radio outbursts of SS Cygni, MNRAS 460 (2016) 3720-3732.

[38] D. L. Coppejans, et al., Dwarf nova-type cataclysmic variable stars are significant radio emitters, MNRAS 463 (2016) 2229-2241.

[39] E. G. Körding, C. Knigge, T. Tzioumis, and R. Fender, Detection of radio emission from a nova-like cataclysmic variable: evidence of jets?, MNRAS 418 (2011) L129-L132.

[40] D. L. Coppejans, E. G. Körding, J. C. A. Miller-Jones, M. P. Rupen, C. Knigge, G. R. Sivakoff, and P. J. Groot, Novalike cataclysmic variables are significant radio emitters, MNRAS 451 (2015) 3801-3813.

[41] R. P. Fender, K. Southwell, and A. K. Tzioumis, A radio survey of supersoft, persistent and transient X-ray sources in the Magellanic Clouds, MNRAS 298 (1998) 692-696.

[42] S. Potter, et al., A new two channel high-speed photo-polarimeter (HIPPO) for the SAAO, in Ground-based and Airborne Instrumentation for Astronomy II, vol. 7014 of Proceedomgs of the SPIE, p. 70145E, 2008.

[43] SALT Ast Ops, Proposal Information for SALT Call for Proposals: 2016 Semester 2. SALT, 2016. Version 1.0. Available at:

pysalt.salt.ac.za/proposal_calls/current/Proposalcall.pdf (accessed 18-12-2016).

[44] A. A. Abdo, et al., Gamma-Ray Emission Concurrent with the Nova in the Symbiotic Binary V407 Cygni, Science 329 (2010) 817-821.

[45] M. Ackermann, et al., Fermi establishes classical novae as a distinct class of gamma-ray sources, Science 345 (2014) 554-558.

[46] C. C. Cheung, et al., Fermi-LAT Gamma-Ray Detections of Classical Novae V1369 Centauri 2013 and V5668 Sagittarii 2015, ApJ 826 (2016) 142.

[47] The Fermi-LAT Collaboration, Fermi establishes classical novae as a distinct class of gamma-ray sources, Science, 345 (2014) 554

[48] B. D. Metzger, T. Finzell, I. Vurm, R. Hascoët, A. M. Beloborodov, and L. Chomiuk, Gamma-ray novae as probes of relativistic particle acceleration at non-relativistic shocks, MNRAS $\mathbf{4 5 0}$ (2015) 2739-2748.

[49] D. W. Hoard, et al., Nova-like Cataclysmic Variables in the Infrared, ApJ 786 (2014) 68.

[50] A. T. Araudo and L. F. Rodríguez, Jets from massive protostars as gamma-ray sources: The case of IRAS 18162-2048, in American Institute of Physics Conference Series (F. A. Aharonian, W. Hofmann, and F. M. Rieger, eds.), vol. 1505 of American Institute of Physics Conference Series, pp. 281-284, 2012.

[51] M. Actis, et al., Design concepts for the Cherenkov Telescope Array CTA: an advanced facility for ground-based high-energy gamma-ray astronomy, Experimental Astronomy 32 (2011) 193-316.

[52] J. Sitarek and W. Bednarek, GeV-TeV gamma rays and neutrinos from the Nova V407 Cygni, PhRvD 86 (2012) 063011. 